\title{
Functional Recovery of Axonal Injury Induced by Gelatin-Hydrogel Film and PRP: An Initial Study in Rats
}

\author{
Retno Ardhani' ${ }^{1}$, Rina Susilowati ${ }^{2}$, Ika Dewi Ana ${ }^{*}$ \\ ${ }^{1}$ Dental Biomedical Sciences Department, Faculty of Dentistry, Universitas Gadjah Mada, Yogyakarta, Indonesia \\ ${ }^{2}$ Histology and Cell Biology Department, Faculty of Medicine, Universitas Gadjah Mada, Yogyakarta, Indonesia \\ Email: ikadewiana@ugm.ac.id
}

Received 20 December 2014; accepted 16 March 2015; published 17 March 2015

Copyright (C) 2015 by authors and Scientific Research Publishing Inc.

This work is licensed under the Creative Commons Attribution International License (CC BY). http://creativecommons.org/licenses/by/4.0/

(c) (i) Open Access

\begin{abstract}
In today's society, axonal regeneration is considered very important because of high incidence of nerve injuries. The injury may cause vast damage of extracellular matrix leading to the condition wherein cell lost their environment to live. In this situation, new strategies by the introduction of scaffold to function as synthetic extracellular matrix are needed. In the field of tissue engineering, a large variety of carriers and matrices have been utilized as a scaffold to direct axonal growth. In this study, a new strategy by the combined application of gelatin hydrogel film and Platelet Rich Plasma (PRP) to induce functional recovery of sciatic nerve crushed injury was investigated in Wistar rat model. Assessment of motorist and sensory functions were done to evaluate the recovery processes. It was found that the application of gelatin hydrogel film with controlled release manner combined with PRP results faster axonal regeneration. Further research by different injury model and other investigation factors are needed to confirm the effectiveness of the strategy.
\end{abstract}

\section{Keywords}

Tissue Engineering, Functional Recovery, Axonal Injury, Gelatin Hydrogel, PRP

\section{Introduction}

In the area of medicine and dentistry, for example, tooth extraction, implant treatment, and reconstructive surgery may cause axonal injury. When axonal injury happens, it is considered as serious health problems, leading to life-long disability or permanent impairment. Although spontaneous regeneration is rarely occurs in axon, its

\footnotetext{
${ }^{*}$ Corresponding author.
} 
regenerative potential is basically high. Therapy to repair damaged axon has been extensively developed, but full functional recovery is still difficult to achieve [1]-[3].

Surgical reconnection, autologous graft and devices application are among therapies indicated for small axonal injury. Meanwhile, in larger defect autologous graft may be applied but it needs multiple surgeries remaining defect on donor sites [4]. This is because vast damage of extracellular matrix happens in large defect leading to the condition wherein cell lost their environment to live. In this situation, new strategies by the introduction of scaffold to function as synthetic extracellular matrix are needed [5].

In the field of tissue engineering, a large variety of carriers and matrices have been utilized as a scaffold to direct axonal growth, widely known as entubulation method, by minimizing cellular and humoral factors suppressing nerve regeneration in the environment. Some conduits made of various biomaterials have been being developed and tested in promoting regeneration [1] [2] [6]. In this context, gelatin is known to be good bio-polymer candidate that can be designed to form conduit. The advantage of the use of gelatin hydrogel, apart from the three dimensional (3D) structure provided to the cells embedded, is that material properties can be adapted including adhesiveness, stiffness, cell signaling potential, size and shape. Hydrogel film from gelatin is also easy to be prepared and its safety has been proven. Haraguchi et al. investigated the application of gelatin hydrogel membrane and found it promising vein graft when combined with basic-Fibroblast Growth Factor (b-FGF) [7]. A lot of research also proved that gelatin hydrogel microsphere promoted better facial nerve recovery after compressive injury when it was combined with b-FGF [2] [6]-[9].

Besides the utilization of scaffold, to date most attention has been given to growth factors including b-FGF to induce tissue regeneration. However, the half-life of the growth factors, the complexity of recombinant technique to prepare growth factors and the cost of it remain problems [4]. These issues lead to the use of Platelet Rich Plasma (PRP) which proved to promote axonal sprouting and myelin development. Since PRP is easy to be collected from patient's blood using uncomplicated technology, the feasibility of its application is better compared to recombinant growth factors. In general, PRP is defined as an autologous concentration of platelets in a small volume of plasma [10]. It is considered to be a rich sources of platelet derived growth factor (PDGF), transforming growth factor beta (TGF- $\beta$ ), vascular endothelial growth factor (VEGF), epithelial growth factor (EGF) and b-FGF [10] [11].

The focus of this study is on tissue engineering approach to axonal regeneration as one among high incidence in today's society [12]. In this study, a new strategy by the combined application of gelatin hydrogel film and PRP to induce functional recovery of sciatic nerve crushed injury was investigated in Wistar rat model. Assessment of motorist and sensory functions were done to evaluate the recovery processes.

\section{Materials and Methods}

\subsection{Gelatin-Hydrogel Preparation}

Gelatin-hydrogel used in this study was prepared from 5 wt\% Type B gelatin of bovine bone (Nitta Gelatin, Japan). The gelatin-hydrogel was poured into polyprophylene mold $\left(4 \times 4 \mathrm{~cm}^{2}\right.$, Balance dish, BioBik, Japan) and kept for 4 days in $4^{\circ} \mathrm{C}$ refrigerator to obtain dried gelatin film. Dehydrothermal treatment to crosslink gelatin-hydrogel film was done at $140^{\circ} \mathrm{C}$ for 48 hours. To control the fabrication of the film, tensile strength of the film was assessed using Universal Testing Machine (Zwick, Germany) in a $10 \mathrm{~mm} / \mathrm{min}$ crosshead speed on dried condition. Furthermore, the microstructure was randomly assessed by Scanning Electron Microscopy (Hitachi, Japan). Meanwhile, swelling behavior of the film was calculated from film weight before and after 24 hours embedding in phosphate buffered saline (PBS) of $7.4 \mathrm{pH}$.

\subsection{Animal Model}

The protocol for this study was approved by Medical and Health Research Ethics Committee of the Faculty of Medicine, Universitas Gadjah Mada (Reference Number KE/FK/55/EC dated 18/01/2012). National guidelines for the care and use of laboratory animals were applied during the study. The animals were housed in cages located at the Integrated Research and Testing Laboratory (LPPT), Universitas Gadjah Mada. Fifty-two (52) male Wistar rats (age 3 months, weight $248.67 \pm 17.33 \mathrm{~g}$ ) were randomly divided into four experimental groups: M0P0 (crush injury), M1P0 (film), M0P1 (PRP) and M1P1 (film with PRP) groups. Functional evaluation was done at Days 3, 7, 14 and 21 after surgery by 4 blinded operators (2 operators for each sensory and motor test). 


\subsection{Coctail of Growth Factors Preparation}

Platelet Rich Plasma as an autologous growth factor was prepared from rat blood. After sedated using intra muscular 80 - $100 \mathrm{mg} / \mathrm{kg}$ weight ketamine hydrochloride injection, retro orbital veins blood was obtained using plain haematocrit tube (Micro-Haematocrit-tubes, Assistent). Peripheral blood was double-centrifugated using 1000 and 2100 G velocity. Platelet count was conducted before and after centrifugation in Clinical Pathology Department, Faculty of Medicine, Universitas Gadjah Mada. Activation using $10 \% \mathrm{CaCl}_{2}$ was done on PRP before application to result PRP gel.

\subsection{Crush Injury Model}

After sedated using intra muscular 80 - $100 \mathrm{mg} / \mathrm{kg}$ weight ketamine hydrochloride with xylazine 5 - $10 \mathrm{mg} / \mathrm{kg}$ weight injection, right hind limb of each subject was shaved and sprayed by $70 \%$ ethanol as antiseptic. Surgical blade was used to incise the skin and maximus gluteus muscle. Dressing forceps and retractor were then used to visualize sciatic nerve. Injury was made by crushing the sciatic nerve using non-serrated clamp at $0.20 \mathrm{MPa}$ for 60 seconds in $2.5 \mathrm{~mm}$ length after suturing the nerve epineurium in distal part of crush injury area (8-O black nylon suture, Bear, Japan). Table 1 shows grouping of the rats.

\subsection{Scaffold and Coctails of Growth Factor Application}

Application of either gelatin hydrogel film, PRP or both was conducted after suturing the nerve epineurium. Gelatin hydrogel film was prepared in $0.5 \times 1 \mathrm{~cm}^{2}$ dimension and sterilized using dry Ethylene Oxide Gas (EOG) method. Gelatin hydrogel film was wrapped on the crushed injury area and PRP (in $210 \mathrm{~mL}$ added by $30 \mathrm{~mL}$ $\mathrm{CaCl}_{2}$ ) was applied directly upon the area. In combined application, PRP was applied after gelatin hydrogel film was placed under crushed sciatic nerve then it was wrapped to cover the area. Skin and muscle were repositioned carefully and irrigated using antibiotic solution. The skin was sutured (4-O black nylon suture, Bear, Japan) and $10 \%$ povidone iodine as an antiseptic was applied upon surgical wound (Betadine, Jakarta, Indonesia). Antibiotic and analgesia were given twice a day for three days after surgical procedure (ad libitum).

\subsection{Functional Recovery Test}

Walking Track Analysis and Nociceptive Withdrawal Response Test as functional test applied in this study used normal limb on the same rat individual as negative control. The 3R principle in the use of animal model (especially to reduce number of rats) was applied during the study by testing normal limb of the same rat individual. Functional recovery of sciatic nerve was evaluated at Days 3, 7, 14 and 21. Nociceptive withdrawal reflex test was conducted to evaluate sensory function recovery. For nociceptive withdrawal reflex test, subject was positioned to stand at one hind limb on hot plate and the other on room temperature. The position was shifted after 15 seconds recovery phase and reproduced for 5 times. Nociceptive withdrawal reflex in 4 seconds or less when tested at $56^{\circ} \mathrm{C}$ hot plate was considered as normal.

Walking track analysis was used to examine motor function recovery. Rat was allowed to walk along walking track analysis apparatus lane after permanent marker ink (Snowman, Japan) application on hind limb to produce

\begin{tabular}{|c|c|c|c|c|}
\hline Termination Day & Crush (M0P0) & Crush + Film (M1P0) & Crush + PRP (M0P1) & Crush + Film + PRP (M1P1) \\
\hline 7 & 4 & 4 & 4 & 4 \\
\hline 14 & 4 & 4 & 4 & 4 \\
\hline 21 & 4 & 4 & 4 & 4 \\
\hline Sub-Total by Group & 12 & 12 & 12 & 12 \\
\hline Total & & & Rats & \\
\hline
\end{tabular}

\footnotetext{
${ }^{*} 4$ Normal individual rats without treatment were used as a reference for functional recovery test.
} 
a trace upon white paper on the lane. Five traces were made from each subject on each assessment time. The injured leg was compared to the non-injured leg from the same individual as the control. Blinded observer recorded the presence or absence of inability to lift foot entirely from the ground (foot-dragging), inability to spread the toes and to measure the paw length. Quantitative measurement was conducted using Sciatic Functional Index (SFI) counted from traces measurement according to Bain-Mackinnon-Hunter formula [13]-[15]. The traces measurement includes distance from the heel to the third toe (Paw Length or PL), distance between the first and the fifth toe (Toe Spread or TS) and distance from the second to the fourth toe (Intermediary Toe Spread or ITS). Zero value of SFI showed normal function and -100 value showed total impairment.

$$
\mathrm{SFI}=-38.3\left(\frac{\mathrm{EPL}-\mathrm{NPL}}{\mathrm{NPL}}\right)+109.5\left(\frac{\mathrm{ETS}-\mathrm{NTS}}{\mathrm{NTS}}\right)+13.3\left(\frac{\mathrm{EIT}-\mathrm{NIT}}{\mathrm{NIT}}\right)-8.8
$$

SFI: Sciatic Functional Index; EPL: Experimental Paw Length; NPL: Normal Paw Length; ETS: Experimental Toe Spread; NTS: Normal Toe Spread; EIT: Experimental Intermediary Toe Spread; NIT: Normal Intermediary Toe Spread.

\subsection{Histological Examination}

Axonal growth determination was conducted by calculating axonal density per $1 \mu \mathrm{m}^{2}$ area on histological preparation of each rat on 7, 14 and 21 days after surgery. On the day of termination, rats were sedated using 100 $\mathrm{mg} / \mathrm{kg}$ weight ketamine hydrochloric with 5 - $10 \mathrm{mg} / \mathrm{kg}$ weight intramuscularly and perfused with $4 \%$ paraformaldehyde and $1 \%$ glutaraldehyde in $\mathrm{pH}$ 7.4 PBS fixatives. Transected nerve was harvested and immersed in the same fixative for $72 \mathrm{~h}$ in $4^{\circ} \mathrm{C}$ followed by graded alcohol dehydrating and paraffin embedding. The $6 \mu \mathrm{m}$ sectioned tissue was stained using $0.1 \%$ Tuloidine Blue in aqueous solution. Histological examination was conducted by two independent blinded observers with agreement among them. Distal region next to bifurcation was used to quantify axonal density.

\section{Results}

\subsection{Properties of the Gelatin Hydrogel Film}

Table 2 shows the properties of the gelatin hydrogel used in this study. Gelatin hydrogel films prepared in this study showed the percentage of $76.00 \pm 2.78$ water content after being embedded in PBS for 24 hours. The tensile strength of the gelatin hydrogel film was $37.18 \pm 2.14 \mathrm{MPa}$. Figure 1 shows homogenous microstructure of the gelatin hydrogelfilm prepared in the study. There was variability of the weight of the films due to variation during manual moldings. The average weight of the films applied in this study was $1.68 \pm 0.13 \mathrm{mg}$ (M1P0) and $1.70 \pm 0.14 \mathrm{mg}$ (M1P1). Film weight was known to be normally distributed as shown by the result of F-test (F calculation 1.27; $p>0.05$ ) and no significant differences between groups, as shown by the result of $\mathrm{T}$ test (Tcalculation $0.01 ; p>0.05$ ).

\subsection{Platelet Number}

Whole blood for preparing PRP was obtained from M0P1 and M1P1 group of animal model on the day of the treatment. Whole blood derived from each rats was individually processed to be able to prepare autologous PRP. In order to confirm platelet concentration, platelet count was done before and after double centrifugations, as shown in Table 3. Statistical analysis on platelet number using Mann-Whitney method showed no significant

Table 2. Properties of gelatin-hydrogel film used in the study.

\begin{tabular}{cc}
\hline Properties & Value \\
\hline Water content (\%) & $76.00 \pm 2.78$ \\
Tensile strength (MPa) & $37.18 \pm 2.14$ \\
& \\
Weight (mg) & $1.68 \pm 0.13$ (M1P0 Group) \\
& $1.70 \pm 0.14$ (M1P1 Group) \\
\hline
\end{tabular}




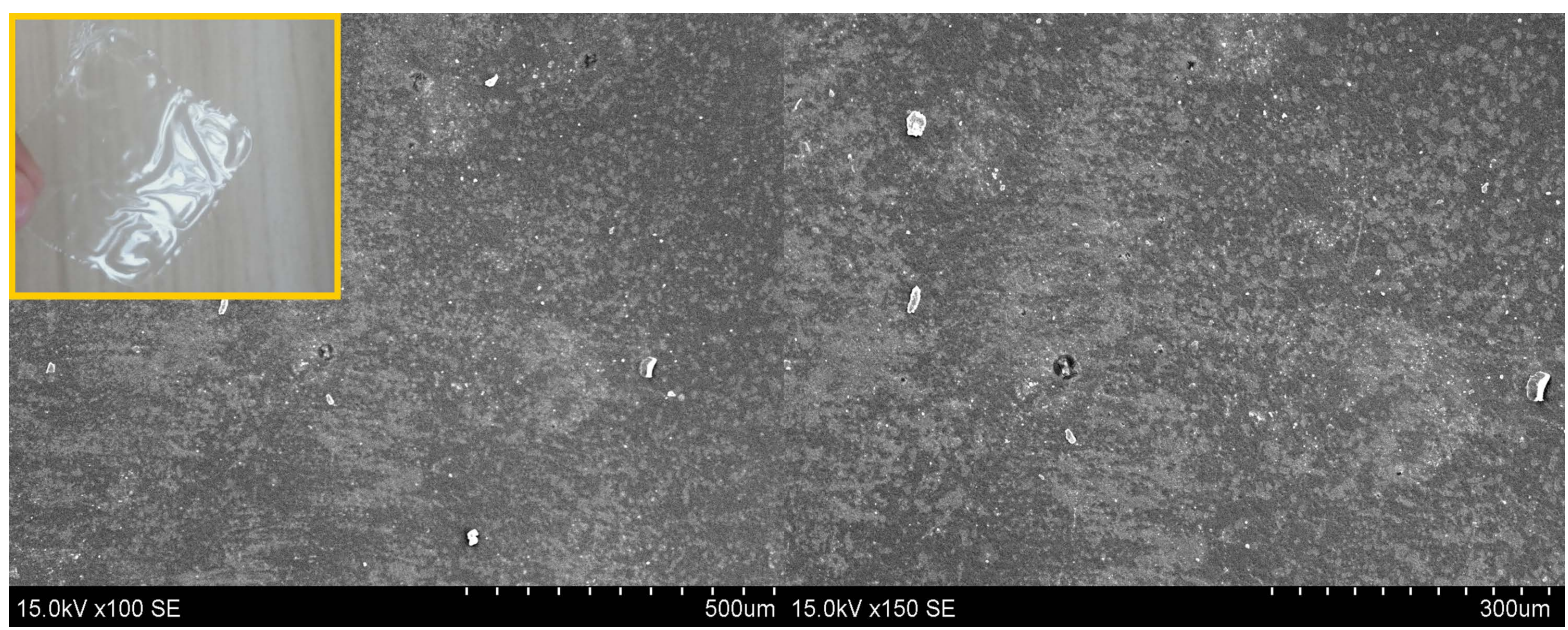

Figure 1. Macrostructure and microstructure of gelatin-hydrogel film used in the study, showing homogenous structure of gelatin hydrogel film.

Table 3. Number of platelet used in the study (per $1 \mu \mathrm{L}$ peripheral blood).

\begin{tabular}{ccc}
\hline Group & Before & After \\
\hline M0P1 & $630923.08 \pm 197.16$ & $661866.78 \pm 108.06$ \\
M1P1 & $1553308.00 \pm 569.37$ & $1,614,933 \pm 583.97$ \\
\hline
\end{tabular}

difference on the applied PRP between M0P1 and M1P1 groups $(p<0.05)$. Based on this result, it was assumed that platelet number of PRP applied in this study was considered well controlled.

\subsection{Sensory Function Recovery}

Nociceptive withdrawal reflex performed by each rat was recorded as normal and abnormal, defined by $4 \mathrm{~s}$ threshold. Withdrawal reflex recorded after $4 \mathrm{~s}$ showed abnormality of sensory function. In this study, sensory function of all rats was abnormal until 21 days of observation. Figure 2 describes the measurement process of walking track paper and Figure 3 describes nociceptive withdrawal reflex (NWR) during 21 days observation. Table 4 shows normal value as a reference for NWR. The M1P1 group showed the most noticable changes along treatment period. Kruskal-Wallis test showed that the changes was significantly different (K calculated $100.30 ; p<0.05$ ) and Tukey test showed that the median nociceptive withdrawal reflex test result was not significanly different from predicted order (Day $21<14<3<7$; Z calculated 1.95).

\subsection{Motor Function Recovery}

Gait observation showed that no rat presented foot dragging at all examination days. Inability to spread the toes was observed on all rats at Days 3 to 14. At Day 21 all rats showed ability to spread the toes and their traces started to be measurable. Score of SFI derived from Walking Track Analysis of M0P0, M1P0, M0P1 and M1P1 is shown on Table 5, completed by Table 6 for a reference in normal rats at Day 21.

\subsection{Histological Structure}

As shown on Table 7, axonal density in M0P0 group decreased from Day $7\left(0.73 / 1000 \mu m^{2}\right)$ to $14\left(0.44 / 1000 \mu m^{2}\right)$ and increased after Day $21\left(0.53 / 1000 \mu \mathrm{m}^{2}\right)$. Similar pattern was found in M1P1 group: 0.87/1000 $\mu \mathrm{m}^{2}$ at Day 7, $0.62 / 1000 \mu \mathrm{m}^{2}$ at Day 14 , and $0.81 / 1000 \mu \mathrm{m}^{2}$. However, axonal density in M1P0 and M0P1 group were decreased along observation periods. At Day 21, M1P1 group showed the highest axonal density among all treatment group ( $\mathrm{K}$ calculated $-1.94, p>0.05$ ). Figure 4 shows highest axonal density found in the M1P1 group. 


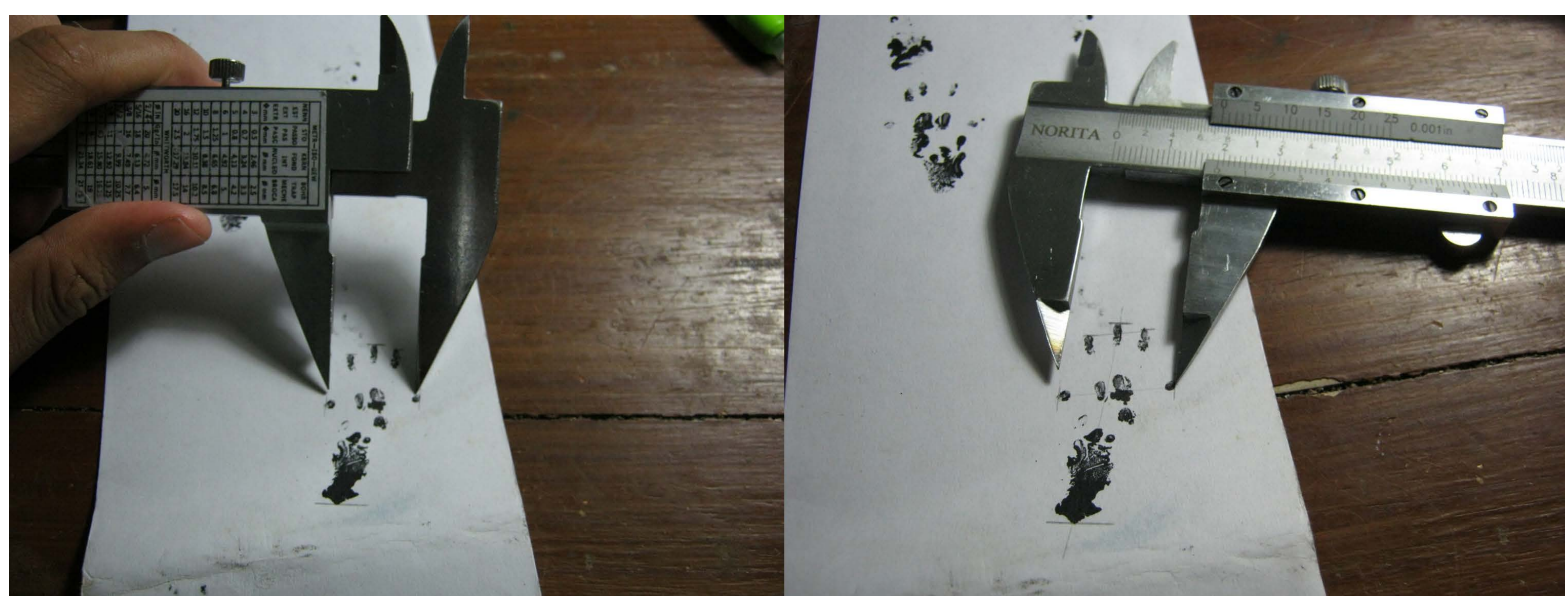

Figure 2. Measurement of walking track paper.

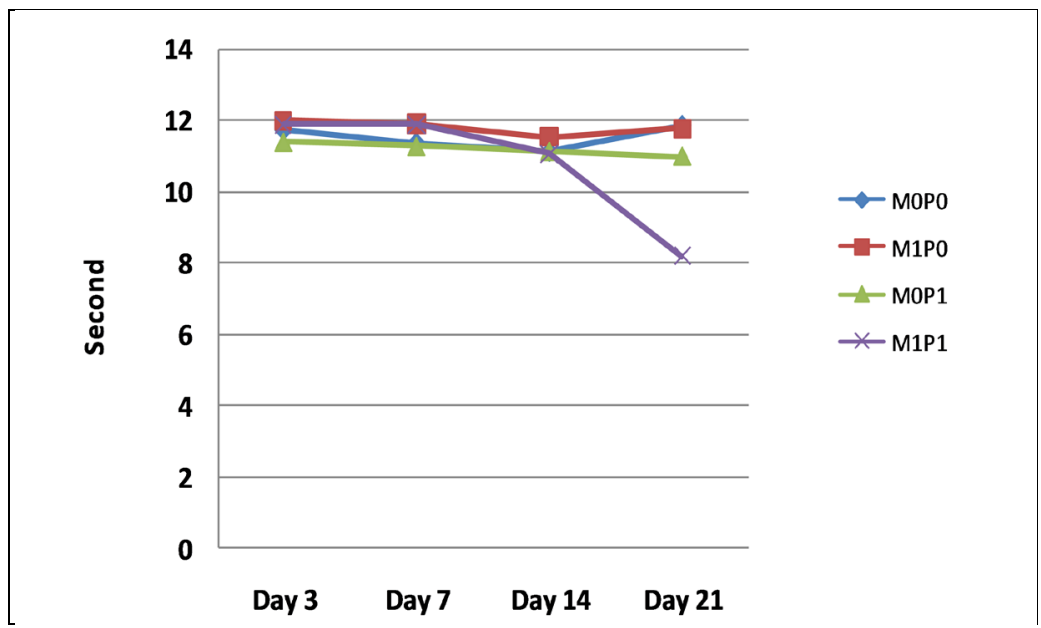

Figure 3. Nociceptive withdrawal reflect on Day 21.

Table 4. Results of NWR (Nociceptive Withdrawal Reflect) in normal rats at Day 21.

\begin{tabular}{ccc}
\hline Normal Rat & NWR (in second) \\
\hline Rat 1 & 3.6 \\
Rat 2 & 2.0 \\
Rat 3 & 1.8 \\
Rat 4 & 4.0 \\
Mean \pm SD & $\mathbf{2 . 8 5} \pm \mathbf{1 . 1 2}$ \\
\hline
\end{tabular}

Table 5. Motor function recovery by means of SFI (Sciatic Function Index) from Days 3 to 21. Normal limb was applied as control.

\begin{tabular}{ccccc}
\hline Group & Day 3 & Day 7 & Day 14 & Day 21* $^{*}$ \\
\hline Crush injury (M0P0) & Inability to spread toes & Inability to spread toes & Inability to spread toes & $-26.29 \pm 7.09$ \\
Film (M1P0) & Inability to spread toes & Inability to spread toes & Inability to spread toes & $31.74 \pm 12.68$ \\
PRP (M0P1) & Inability to spread toes & Inability to spread toes & Inability to spread toes & $-30.74 \pm 30.34$ \\
Film-PRP (M1P1) & Inability to spread toes & Inability to spread toes & Inability to spread toes & $-21.29 \pm 22.12$ \\
\hline
\end{tabular}

${ }^{*}$ In scoring system. Normal motor function falls at 0 score and the variation ranges from +11 to -11 , meanwhile -100 means complete motor function disability [22]. 
Table 6. Results of SFI (Sciatic Functional Index) in normal rats at Day 21.

\begin{tabular}{ccc}
\hline Normal Rat & SFI \\
\hline Rat 1 & -2.27 \\
Rat 2 & 5.85 \\
Rat 3 & 12.60 \\
Rat 4 & 9.04 \\
\hline
\end{tabular}

Table 7. Axonal density in $1000 \mu \mathrm{m}^{2}$ on Days 7, 14 and 21 in the distal part to crush injury site.

\begin{tabular}{cccc}
\hline Group & Day 7 & Day 14 & Day 21 \\
\hline Crush injury (M0P0) & $0.73 \pm 0.06$ & $0.44 \pm 0.20$ & $0.53 \pm 0.11$ \\
Film (M1P0) & $0.92 \pm 0.16$ & $0.64 \pm 0.21$ & $0.61 \pm 0.16$ \\
PRP (M0P1) & $0.86 \pm 0.13$ & $0.73 \pm 0.19$ & $0.67 \pm 0.24$ \\
Film-PRP (M1P1) & $0.87 \pm 0.14$ & $0.62 \pm 0.18$ & $0.81 \pm 0.28$ \\
\hline
\end{tabular}

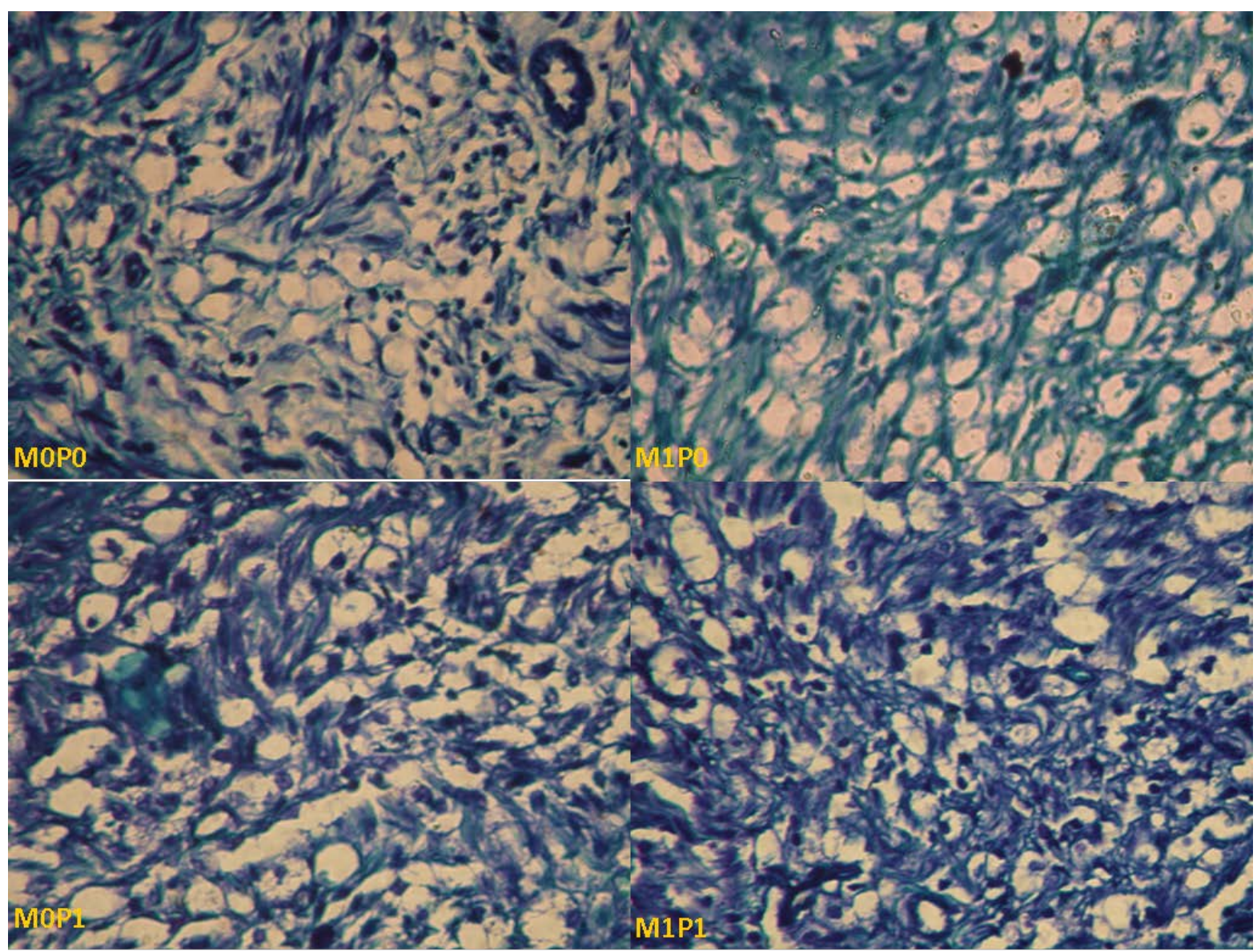

Figure 4. Axonal density after 21 days in (a) M0P0 group; (b) M1P0 group; (c) M0P1 group; and (d) M1P1 group. Highest axonal density was found in $\mathrm{D}$. 


\section{Discussion}

Platelet-Rich Plasma (PRP) has been widely known as a source of variable autologous growth factors, but these growth factors are soluble and easily diminished from the site where it is applied before showing its therapeutic effects. In this research, gelatin hydrogel films were used to maintain autologous growth factors released by PRP on crush injury area by absorbing early secreted platelet growth factors and releasing it in controlled manner. It was reported that platelets are able to secret $70 \%$ of their stored growth factors within 10 minutes after activation [10]. It was also reported that platelets have an ability to produce additional amounts of growth factors for about 8 days until they are depleted and die [10]. Thus, the application of gelatin hydrogel film was expected to increase growth factor concentration during sciatic nerve regeneration.

Since PRP is easy to be collected from patient's blood using simple technology, the feasibility of its application is better compared to the application of recombinant growth factors. It is considered that PRP is a rich sources of platelet derived growth factor (PDGF), transforming growth factor beta (TGF- $\beta$ ), vascular endothelial growth factor (VEGF), epithelial growth factor (EGF), and b-FGF [10] [11].

In this research double centrifugation method as suggested by Marxwas used to prepare plasma containing more than $1,000,000$ platelet/ $\mu$ l or PRP [10]. Platelet number of PRP in this study was increasing up to about 250\% (about 1.5 million platelet/ $\mu$ l). The increase of platelet number in this study was lower than the one reported by Messora et al. [16]. They found the increase of platelet number up to 390\% higher (about 2.6 million platelet/ $\mu \mathrm{l}$ ) than peripheral blood using centrifugation velocity of $160 \mathrm{G}$ and $400 \mathrm{G}$. Higher centrifugation power used in this study (1000 G and $1600 \mathrm{G}$ ) may result into the damage on platelet membrane [16]. However, referring to the study done by Plachokova et al. [17], lower centrifugation power (800 rpm or $<160 \mathrm{G}$ ) to prepare PRP from rat blood did not result higher platelet number (300\% higher) than that of the one did by Messora and group (390\% higher) [16].

Due to variability of PRP preparation methods, comparing the effect of PRP in this study with other studies can not be precisely performed. On the other hand, studies on PRP application to enhance peripheral nerve regeneration were considered very limited. Furthermore, systematic review on peripheral nerve regeneration treatment using PRP is not available. Plachokova et al. [17] suggested that detailed description of the PRP preparation protocol on each study is required to perform comparation between studies, including information about cells separator used, velocity and steps of centifugation, amount of blood collected before PRP preparation, amount of PRP and its platelet concentration, and coagulation promoters (including name, doses, mixing ratio of PRP). Thus, the PRP preparation method used in this study was considered acceptable method to result $250 \%$ higher platelet number.

Reports on PRP safety is very limited but studies showed that no clinical sign of PRP negative effect [17]. Clinical uses of PRP have been reported, such as in graft incorporation of mandibular reconstruction patient, periodontal diseases treatment, cosmetic surgery including face or neck lift and breast modification, chronic ulcer, and in enhancing surgical wound healing. However, treatment-related complication have not been reported yet [17]-[19].

In this study, functional recovery was achieved in all groups, even in the group without treatment (M0P0). The study used 0.20 MPa pressure of non-serrated clamp applied for 60 second, promoted repairable damage on sciatic nerve which is considered as axonotmesis. Axonotmesis is defined as an injury where axons are damaged but nerve sheaths remain intact. Although the pressure applied in the study was considered very high compared to other studies, it was found that the results were in agreement with the previous studies related to the phenomena of axonotmesis, as shown on Table 8. Axonotmesis is defined as an injury where axons are damaged but nerve sheaths remain intact. Muir [3] mentioned that functional recovery can be achieved well in axonotmesis without medical intervention. In this study also the approach by application of gelatin hydrogel film only (M1P0)

Table 8. Nerve injury model used in the previous studies.

\begin{tabular}{llcr}
\hline \multicolumn{1}{c}{ Authors } & Model & Type of Injury & Reference Number \\
\hline Oliviera et al. $(2001)$ & Non-serrated clamp, $500 \mathrm{~g}, 1000 \mathrm{~g}$, and $1500 \mathrm{~g}, 5 \mathrm{~mm}, 10 \mathrm{~min}$ & Axonotmesis \\
Shirosaki et al. (2009) & Non-serrated clamp, $54 \mathrm{~N}, 30 \mathrm{~s}$ & Axonotmesis \\
Komobuchi et al. $(2010)$ & Micro needle forceps, $1 \mathrm{~mm}, 15.7 \mathrm{~Pa}, 10 \mathrm{~min}$ & Axonotmesis \\
\hline
\end{tabular}


was found to be effective since the gelatin hydrogel film can act as an entubulation to provide proper micro environment for the injured nerve. Meanwhile, in the group of MOP1, localized application of PRP (even without gelatin hydrogel film) may promote faster regeneration. In view of this, proper model of nerve injury is needed to further observe whether a new strategy used in M1P1 model is effective. Crush injury may not be proper model to investigate the effectiveness of a new approach by tissue engineering, and most probably cut nerve model will be an adequate model for future observations.

Although Nociceptive Withdrawal Reflects showed abnormality up to Day 21 in all groups, but it was found that the M1P1 group showed the most noticable changes along treatment period. Walking Track Analysis resulted into SFI also showed the same phenomena wherein M1P1 clearly showed SFI range close to normal functional recovery, compared to other groups. The result of histological study showed the same phenomena with the functional test results, where M1P1 group showed the highest axonal density among all treatment groups. However, it is considered that longer observation period is needed.

Furthermore, this study confirmed gelatin hydrogel safety when it is used for biomedical purposes as suggested by Yamamoto and Tabata [8]. Clinical trials also showed the success of gelatin hydrogel application in tissue engineering in patients with limb ischemia [20] and Sudden Sensorineural Hearing Loss [21]. Based on the study by Wall et al. and Zimmerman [22] [23] pain behaviour in animal following nerve injury can be shown as hyperalgesia and allodynia. In this study, hyperalgesia was not observed during the study and allodynia test was not conducted.

\section{Conclusion}

It was concluded that tissue engineering approach by the application of gelatin hydrogel film combined with PRP resulted faster functional recovery of axonal injury. The axonal density was found highest in the group treated with gelatin hydrogel film combined with PRP. Proper injury model is needed, e.g. with more extreme model such as cut nerve, to further observe whether a new strategy is really effective.

\section{Acknowledgements}

This study was funded by Research Grant of the Institute for Research and Community Services Universitas Gadjah Mada, contract number LPPM-UGM/852/BID.I/2011. Authors thank Professor Yasuhiko Tabata and Professor Masaya Yamamoto of the Institute for Frontier Medical Sciences, Kyoto University, Kyoto, Japan for the knowledge on the basic principle of hydrogel technology and partnership in research capacity building of the Department of Biomedical Sciences, Faculty of Dentistry, Universitas Gadjah Mada, Yogyakarta, Indonesia.

\section{References}

[1] Belkas, J.S., Schoichet, M.S. and Midha, R. (2004) Peripheral Nerve Regeneration through Guidance Tubes. Neurogical Research, 26, 1-10.

[2] Shirosaki, Y., Lopes, M.A., Santos, J.D., Tsuru, K., Hayakawa, S., Osaka, A., Mauricio, A.C. and Geuna, S. (2009) Nerve Regeneration Guided by Chitosan Hybrid Membranes. Archieves of BioCeramics Research, 9, 147-150.

[3] Muir, D. (2010) The Potential of Peripheral Nerve Sheaths in Regeneration and Repair. J Exp Neurol, 223, $102-111$.

[4] Schmidt, C.E. and Leach, J.B. (2003) Neural Tissue Engineering: Strategies for Repair and Regeneration. Annual Review of Biomedical Engineering, 5, 293-347. http://dx.doi.org/10.1146/annurev.bioeng.5.011303.120731

[5] Ikada, Y. (2006) Tissue Engineering: Fundamentals and Applications. In: Hubbard, A., Ed., Interface Science and Technology, Elsevier, Amsterdam.

[6] Tabata, Y. (2009) Current Status of Regenerative Medical Therapy Based on Drug Delivery Technology. Proceeding of Open Lecture on Translational Research in Tissue Engineering: Experiences and Lessons Learned from Japan, in Conjunction with the 59th Anniversary of Universitas Gadjah Mada, Universitas Gadjah Mada, Yogyakarta.

[7] Haraguchi, T., Okada, K., Tabata, Y., Maniwa, Y., Hayashi, Y. and Okita, Y. (2007) Controlled Release of Basic Fibroblast Growth Factor from Gelatin Hydrogel Sheet Improves Structural and Physiological Properties of Vein Graft in Rat. Arteriosclerosis, Thrombosis, and Vascular Biology 27, 548-555. http://dx.doi.org/10.1161/01.ATV.0000254811.11741.2b

[8] Yamamoto, M. and Tabata, Y. (2006) Tissue Engineering by Modulated Gene Delivery. Advanced Drug Delivery Reviews, 58, 535-554. http://dx.doi.org/10.1016/j.addr.2006.03.003

[9] Komobuchi, H., Hato, N., Teraoka, M., Waisaka, H., Takahashi, H., Gyo, K., Tabata, Y. and Yamamoto, M. (2010) 
Basic Fibroblast Growth Factor Combined with Biodegradable Hydrogel Promotes Healing of Facial Nerve after Compression Injury: An Experimental Study. Acta Oto-Laryngologica, 130, 1-6. http://dx.doi.org/10.3109/00016480902896139

[10] Marx, R.E. (2001) Platelet-Rich Plasma: What Is PRP and What Is Not PRP? Implantology, 10, 225-228.

[11] Roussy, Y., Duchesne, M.P.B. and Gagnon, G. (2007) Activation of Human Platelet-Rich Plasmas: Effect on Growth Factors Release, Cell Division and in Vivo Bone Formation. Clinical Oral Implants Research, 18, 639-648. http://dx.doi.org/10.1111/j.1600-0501.2007.01385.x

[12] Schlosshauer, B., Dreesman, L., Schaller, H.E. and Sinis, N. (2006) Synthetic Nerve Guide Implants in Human: A Comprehensive Study. Neurosurgery, 59, 740-748. http://dx.doi.org/10.1227/01.NEU.0000235197.36789.42

[13] Varejão, A.S.P., Meek, M.F., Ferreira, A.J.A., Patrício, J.A.B. and Cabrita, A.M.S. (2001) Functional Evaluation of Peripheral Nerve Regeneration in the Rat: Walking Track Analysis. Journal of Neuroscience Methods, 108, 1-9. http://dx.doi.org/10.1016/S0165-0270(01)00378-8

[14] Sarikcioglu, L., Demirel, B.M. and Utuk, A. (2008) Walking Track Analysis: An Assessment Method for Functional Recovery after Sciatic Nerve Injury in Rat. Folia Morphologica, 68, 1-7.

[15] Oliviera, E.F., Mazzer, N., Barbieri, C.H. and Selli, M. (2001) Correlation between Functional Index and Morphometry to Evaluate Recovery of the Rat Sciatic Nerve Following Crush Injury: Experimental Study. Journal of Reconstructive Microsurgery, 17, 69-76. http://dx.doi.org/10.1055/s-2001-12691

[16] Messora, M.R., Nagata, M.J.H., Furlaneto, F.A.C., Dornelles, R.C.M., Bomfim, S.R.M., Deliberador, T.M., Garcia, V.G. and Bosco, A.F. (2011) A Standardized Research Protocol for Platelet Rich Plasma (PRP) Preparation in Rats. RSBO: Revista Sul-Brasileira de Odontologia, 8, 299-304.

[17] Plachokova, A.S., Van den Dolder, J., Van den Beucken, J.J.P. and Jansen, J.A. (2009) Bone Regenerative Properties of Rat, Goat and Human Platelet-Rich Plasma. International Journal of Oral and Maxillofacial Surgery, 38, 861-869. http://dx.doi.org/10.1016/j.ijom.2009.04.009

[18] Martínez-Zapata, M.J., Martí-Carvajal, A., Solà, I., Bolibar, I., Expósito, J.Á., Rodriguez, L. and García, J. (2009) Efficacy and Safety of the Use of Autologous Plasma Rich in Platelets for Tissue Regeneration: A Systematic Review. Transfusion, 49, 44-56. http://dx.doi.org/10.1111/j.1537-2995.2008.01945.x

[19] Nandakumar, K. and Anila, S. (2007) Application of Platelet-Rich Plasma for Regenerative Therapy in Periodontics. Trends in Biomaterials and Artificial Organs, 20, 78-83.

[20] Marui, A., Tabata, Y., Kojima, S., Yamamoto, M., Tambara, K., Nishina, T., Yoshiaki, S., Inui, K., Hashida, T., Yokoyama, S., Onodera, R., Ikeda, T., Fukushima, M. and Komeda, M. (2007) A Novel Approach to Therapeutic Angiogenesis for Patients with Critical Limb Ischemia by Sustained Release of Basic Fibroblast Growth Factor Using Biodegradable Gelatin Hydrogel: An Initial Report of the Phase I-IIa Study. Circulation Journal, 71, 1181-1186. http://dx.doi.org/10.1253/circj.71.1181

[21] Nakagawa, T., Sakamoto, T., Hiraumi, H., Kikkawa, Y.S., Yamamoto, N., Hamaguchi, K., Ono, K., Yamamoto, M., Tabata, Y., Teramukai, S., Tanaka, S., Tada, H., Onodera, R., Yonezawa, A., Inui, K. and Ito, J. (2010) Topical Insulin-Like Growth Factor 1 Treatment Using Gelatin Hydorgels for Glucocorticoid-Resistant Sudden Sensorineural Hearing Loss: A Prospective Clinical Trial. BMC Medicine, 8, 76. http://dx.doi.org/10.1186/1741-7015-8-76

[22] Wall, P.D., Devor, M., Inbal, R., Scadding, J.W., Schonfeld, D., Seltzer, Z. and Tomkiewicz, M.M. (1979) Autotomy Following Peripheral Nerve Lesions: Experimental Anaesthesia Dolorosa. Pain, 7, 103-113. http://dx.doi.org/10.1016/0304-3959(79)90002-2

[23] Zimmerman, M. (2001) Pathobiology of Neuropathic Pain. European Journal of Pharmacology, 429, $23-37$. http://dx.doi.org/10.1016/S0014-2999(01)01303-6 